\title{
Gut Microbiota Profiles in Nonalcoholic Fatty Liver Disease and Its Possible Impact on Disease Progression Evaluated with Transient Elastography: Lesson Learnt from 60 Cases
}

\author{
Winda P. Bastian ${ }^{a} \quad$ Irsan Hasan $^{a} \quad$ C. Rinaldi A. Lesmana ${ }^{a}$ \\ Ikhwan Rinaldi ${ }^{b}$ Rino A. Gani ${ }^{a}$ \\ aHepatobiliary Division, Department of Internal Medicine, Dr. Cipto Mangunkusumo \\ Hospital, Universitas Indonesia, Jakarta, Indonesia; ${ }^{b}$ Clinical Epidemiology Unit and \\ Hematology and Oncology Division, Department of Internal Medicine, Dr. Cipto \\ Mangunkusumo Hospital, Universitas Indonesia, Jakarta, Indonesia
}

\section{Keywords}

Fatty liver · Gut microbiome · Transient elastography

\section{Abstract}

Background: Dysbiosis of the gut microbiota has been considered to have a role in nonalcoholic fatty liver disease (NAFLD) progression. However, there is still lack of studies regarding this phenomenon. Aim: To find the difference in the proportion of gut microbiota in NAFLD patients based on the stages of liver fibrosis. Patients and Methods: A cross-sectional study was conducted at Dr. Cipto Mangunkusumo Hospital, which is the largest tertiary referral center. Human fecal samples from NAFLD patients who came to the outpatient clinic were collected consecutively. The stool sample examination was performed using an isolation DNA kit (Tiangen) and quantitative real-time polymerase chain reaction (Fast 7500). Clinical and 


\section{Case Reports in Gastroenterology}

Case Rep Gastroenterol 2019;13:125-133

(C) 2019 The Author(s). Published by S. Karger AG, Base www.karger.com/crg

Bastian et al.: Gut Microbiota Profiles in Nonalcoholic Fatty Liver Disease

laboratory data were also collected. The stage of fibrosis was diagnosed based on transient elastography (FibroScan ${ }^{\circledR} 502$ Touch; Echosens, France). Results: Of 60 NAFLD human fecal samples, 35 patients had nonsignificant fibrosis and 25 patients had significant fibrosis $(46.7 \%$ male and $53.3 \%$ female; median age 56 years). Most patients had diabetes (85\%), dyslipidemia (58.3\%), obesity (58.3\%), and central obesity (90\%). The proportion of Bacteroides was higher when compared to Lactobacillus and Bifidobacteria. Of these 3 microbiota, the proportion of Bacteroides was significantly higher in the significant fibrosis group when compared to the nonsignificant fibrosis group. Conclusion: There is a change in the composition of gut microbiota in NAFLD patients. The proportion of Bacteroides is significantly higher in significant liver fibrosis, which may play a role in NAFLD progression.

(C) 2019 The Author(s)

Published by S. Karger AG, Basel

\section{Introduction}

Nonalcoholic fatty liver disease (NAFLD) is a liver disease characterized by excessive fat accumulation in the liver, without any other cause of secondary steatosis such as excessive alcohol consumption, use of certain drugs, or hereditary diseases. NAFLD is divided into 2 categories, simple steatosis and NASH (nonalcoholic steatohepatitis). Simple steatosis is defined as the presence of liver fat in the absence of significant injury to the liver cells, whereas NASH is the presence of liver steatosis with significant inflammation and damage to the hepatocytes. NASH could further lead to liver cirrhosis and hepatocellular carcinoma if not well managed $[1,2]$. Prevalence of NAFLD has been increasing along with the number of metabolic diseases. The prevalence of NAFLD in Europe is $20-30 \%$, while the prevalence in Asia is $15-30 \%$, and $30.6 \%$ in Indonesia [3]. Although the overall prevalence of NAFLD has the same presentation, the characteristics of the NAFLD population in Western countries when compared to Asian countries might be different. Obesity is deemed to be higher in Western populations, while type II diabetes mellitus is found to be higher in Asian populations [2].

In clinical practice, it is not always easy to differentiate between simple steatosis and $\mathrm{NASH}$, as most of the routine imaging used is transabdominal ultrasound (US). Even though there are US criteria to stratify between mild, moderate, or even severe steatosis, its impact for liver disease progression is still largely unknown. Liver biopsy is still the most trustable modality with regard to its possible sampling error. Transient elastography (FibroScan) has recently become a popular modality to assess liver disease progression in chronic liver disease. Controlled attenuation parameter (CAP) is the new innovation in transient elastography to differentiate mild, moderate, and severe steatosis and it is more reliable than transabdominal US [4-6].

The pathogenesis of NAFLD is a complex interplay, which includes genetic factors, diet, metabolic syndrome such as obesity, and insulin resistance. Currently, the influence of gut dysbiosis which is also known as a change in the composition of gut bacteria, has been hypothesized to have a role in the pathogenesis of NAFLD from steatosis to liver fibrosis. The relationship between gut dysbiosis in the pathogenesis of NAFLD includes the presence of effects on short-chain fatty acids resulting in steatosis, increased gut permeability, translocation of gut microbiota, and increased endotoxin (lipopolysaccharide), which can lead to activation of inflammatory factors and liver fibrosis [7-9]. Very few studies have explored the role of gut 


\section{Case Reports in Gastroenterology}

Case Rep Gastroenterol 2019;13:125-133

DOI: $10.1159 / 000498946$

(c) 2019 The Author(s). Published by S. Karger AG, Base www.karger.com/crg

Bastian et al.: Gut Microbiota Profiles in Nonalcoholic Fatty Liver Disease

microbiota in NAFLD. A previous study in the USA revealed that the proportion of Bacteroidetes increased in simple steatosis patients and healthy subjects [10], while another study in Chinese showed that the proportion of Bacteroidetes in NASH patients was higher than in healthy populations [11]. This study was supported by other studies, showing that gut microbiota affected the degree of liver fibrosis [12-14]. Based on these data, modulation of gut microbiota through dietary intervention and probiotics (Lactobacillus and Bifidobacteria) might have a role in preventing the progression of NAFLD disease so that it can be part of the management of NAFLD in the future $[2,10,12]$.

Gut microbiota composition has been thought to be dependent on childhood diet type, environment, history of antibiotic use, geographical diet type, and diet change. Therefore, this study aimed to examine the proportion of commensal gut microbiota and its possible impact in NAFLD progression. This research is important since there is still a lack of studies regarding this phenomenon and recent studies have not been well characterized. The characteristics of patients in Indonesia (which represents the biggest Southeast Asian country) also show the race factor and different diets. These data will provide knowledge for NASH prevention and management programs in the future.

\section{Methods}

\section{Study Design}

This was a cross-sectional study with consecutive sampling of NAFLD patients at Dr. Cipto Mangunkusumo Hospital in the period March-July 2018. NAFLD is diagnosed based on transabdominal US with higher liver echogenicity when compared to the kidneys. The inclusion criteria for patients in this study were NAFLD patients, and the exclusion criteria were history of significant alcohol consumption ( $>20 \mathrm{~g} /$ day), evidence of hepatitis B or C infection, histological evidence of other concomitant chronic liver diseases, pregnant women, cirrhosis with and without complications (ascites, variceal bleeding, systemic infection, or hepatocellular carcinoma), history of chronic inflammatory bowel disease or bariatric surgery, or treatment with antibiotics within 1 month before inclusion.

\section{Patient and Public Involvement}

The patients were informed about the study protocol, aim, and benefit of stool examination with regard to the awareness of disease progression that they might have and possible management in the future to prevent disease complications. The patients who agreed to be included in this study were provided with a stool container and to collect their stool samples. After all the data analysis was done, the patients would be informed. Other than these processes, the patients were not involved.

\section{Data Collection}

Data on the demographic and clinical characteristics (gender, age, body mass index) were obtained from the patients who met the study criteria. Moreover, the latest laboratory results of HBsAg, anti HCV, HbA1c, triglycerides, AST, and ALT levels in the past 3 months were also collected. The stool sample examination was performed using an isolation DNA kit (Tiangen) and quantitative real-time polymerase chain reaction (Fast 7500). Quantitative polymerase 


\section{Case Reports in Gastroenterology}

Case Rep Gastroenterol 2019;13:125-133

DOI: $10.1159 / 000498946$

(c) 2019 The Author(s). Published by S. Karger AG, Basel www.karger.com/crg

Bastian et al.: Gut Microbiota Profiles in Nonalcoholic Fatty Liver Disease

chain reaction was examined in the feces of NAFLD patients to calculate the number of gut microbiota (Bacteroides, Lactobacillus, and Bifidobacterium) with species-specific $16 \mathrm{~S}$ rRNA gene primers: Bifidobacterium sp. (F-CGGGTGAGTAATGCGTGACC and R-TGATAGGACGCGACCCCA); Lactobacillus sp. (F-AGCAGTAGGGAATCTTCCA and R-AGCAGTAGGGAATCTTCCA); Bacteroides sp. (F-CCTCCGATGGATAGGGGTT and R-CACGCTACTTGGCTGGTTCAG). The primer design was created using Primer Express version 2.0 (Applied Biosystems) and the specificity was confirmed using the Probe Match program [15]. To assure that these primers were specific and attached to the specific bacteria, the BLAST method was used. The standard DNA for quantitative reference used DR10 (Bifido HN019) with the formula (in the PCR machine). The TE-CAP (Fibroscan ${ }^{\circledR} 502$ Touch; Echosens, France) examination was used to assess the degree of fibrosis of NAFLD patients. The fibrosis group was divided into significant fibrosis (F2-F4) and nonsignificant fibrosis (F0-F1). Nonsignificant fibrosis (F0-F1) has a cutoff $<5.7-7.0 \mathrm{kPa}$, and significant fibrosis (F2-F4) has a cutoff of $\geq 7.0-10.4 \mathrm{kPa}$ [16].

\section{Statistical Analysis}

Data with continuous variables and normal distribution are presented as mean \pm SD and those without normal distribution are presented as the median (minimum-maximum). Bivariate analysis between two groups was performed using the Mann-Whitney test. A $p$ value $<0.05$ was considered statistically significant. The statistical analysis in this study was performed using Statistical Package for the Social Sciences (SPSS) software.

\section{Results}

Of the 60 NAFLD cases, 35 patients had nonsignificant fibrosis and 25 patients had significant fibrosis. The median age was 56 years and 32 patients (53.3\%) were female. Most patient have diabetes mellitus (85\%), followed by dyslipidemia (58.3\%), and obesity (58.3\%); however, central obesity was found in $90 \%$ of patients (Table 1 ).

The proportion of Bacteroides (DNA copy number 483,000/gram feces) was the highest when compared to Lactobacillus (DNA copy number 100,800/gram feces) and Bifidobacteria (DNA copy number 12,110/gram feces). The proportion of Bacteroides was significantly different $(p=0.049$ ) between patients with nonsignificant fibrosis (DNA copy number 73,000 /gram feces) and those with significant fibrosis (DNA copy number 310,000/gram feces). In contrast, the proportion of Bifidobacteria was lower in significant fibrosis (DNA copy number 22/gram feces) when compared to nonsignificant fibrosis (DNA copy number 95/gram feces) ( $p=0.288$ ), while the proportion of Lactobacillus was higher in patients with significant fibrosis (DNA copy number 7,000/gram feces) than in those with nonsignificant fibrosis (DNA copy number 2,050/gram feces) ( $p=0.713)$. The proportions of gut microbiota are presented in Figure 1 and Table 2.

\section{Discussion}

To best of our knowledge, this is the first study in Southeast Asia, where Indonesia represents the biggest country in this region, to evaluate the proportion of microbiota in NAFLD 


\section{Case Reports in Gastroenterology} \begin{tabular}{l|l}
\hline Case Rep Gastroenterol 2019;13:125-133 \\
\hline DOI: 10.1159/000498946 & $\begin{array}{l}\text { @ 2019 The Author(s). Published by S. Karger AG, Basel } \\
\text { www.karger.com/crg }\end{array}$
\end{tabular}

Bastian et al.: Gut Microbiota Profiles in Nonalcoholic Fatty Liver Disease

patients and also its impact related to the degree of liver fibrosis. In this study, several things that might be different between countries or among regions which could affect the amount of microbiota have also been considered such as a history of antibiotic use, diet, age, and metabolic components [7]. Of the 60 subjects in this study, the highest NAFLD population was female $(53.3 \%)$ with an average age of 56 years. This is in accordance with the study presented by Chitturi et al. [17], demonstrating that at the age above 50 years, the incidence of NAFLD in females increased compared to males. This is related to the presence of the menopause, which is associated with increased insulin resistance as a risk factor of NAFLD. Most patients have diabetes mellitus (85\%), dyslipidemia (58.3\%), obesity (68.3\%), and central obesity (95\%). This is in accordance with Loomba et al. [2], which stated that the characteristics of NAFLD patients in Asia are different from those in Western countries, with a higher prevalence of diabetes mellitus compared to obesity.

In this study, we found the proportion of Bacteroides (81\%) was the highest when compared to Lactobacillus (16.9\%) and Bifidobacteria (2\%). The result of this study is consistent with previous studies by Wong et al. $[11,16]$ which stated that there was an increase in Bacteroidetes (67.6\%) when compared to Firmicutes (22.3\%), and Actinobacteria and other microbiota (4.1\%). A study by Zhao et al. [18] also showed that there was a dysbiosis in NASH patients with the most phylum, Bacteroidetes (49.11\%). At the genus level, the proportion of Bacteroides was $23.31 \%(p=0.89)$ and that of Bifidobacteria $0.63 \%(p=0.045)$. Bacteroides as a Gram-negative microbiota produce LPS which is correlated to fibrosis liver. Bacteroides are also associated with an increase in deoxycholic, raffinose, and stachyose acids, which increase the risk of NAFLD, and a decrease in short-chain fatty acids, which also play a role in the pathogenesis of NAFLD [18-20].

Based on stages of the fibrosis group, there was a significant increase in the proportion of Bacteroides in the severe fibrosis group when compared with the mild fibrosis group. In contrast, the proportion of Bifidobacteria was found to be decreased in the severe fibrosis group when compared to mild fibrosis groups, although not significantly, while the proportion of Lactobacillus was greater in the severe fibrosis group than in the mild fibrosis group. This study result is in agreement with a previous study by Loomba et al. [14] which showed more Bacteroidetes phyla in the severe fibrosis group (28.46\%) than in the mild fibrosis group $(23.62 \%)(p=0.57)$. In this study, Actinobacteria was found more in the mild fibrosis group $(2.67 \%)$ than in the severe fibrosis group $(2.02 \%)(p=0.78)$, while the proportion of phylum Firmicutes was increased in the significant fibrosis group when compared to the nonsignificant fibrosis group (58.81 vs. $42.61 \%$, respectively) ( $p=0.015$ ). In this study, Bacteroides vulgatus (Bacteroidetes) and Ruminococcus obeum (Firmicutes) were significant species differences between the two groups of fibrosis.

Another study from Boursier et al. [13] also showed the same abundance of Bacteroides, where the proportion was greater in the severe fibrosis group than in the mild fibrosis group (42.4 vs. $57.8 \%$ ) ( $p=0.018)$. This study result supported the theory that dysbiosis could affect the degree of liver fibrosis. An increase in LPS produced by gut bacteria (Gram negative) can lead to activation of inflammatory factors that contribute to the progression of liver fibrosis. Bacteroides accumulation is also associated with the accumulation of branched-chain fatty acids produced by amino acid fermentation, which is a factor that contributes to insulin resistance as a risk factor for NASH. The decrease in the proportion of Bifidobacteria in the severe fibrosis group is in agreement with previously published literature demonstrating that 
the role of modulation of gut microbiota with probiotics can affect the degree of liver fibrosis. It has also been shown that the administration of probiotics containing Bifidobacteria in NAFLD patients can reduce levels of TNF, CRP, transaminase enzymes, endotoxins, and NASH scores, which contribute to NAFLD progression $[21,22]$. However, in this study there were different results in the proportion of the genus Lactobacillus, where the proportion was greater in the severe fibrosis group than in the mild fibrosis group. This is in contrast to previous studies by both Loomba et al. [14] and Boursier et al. [13], who stated that the proportion of phylum Firmicutes as Gram positive was lower in severe fibrosis. This phenomenon may be due to the different examination methods used, where in previous studies the 16S RNA pyrosequencing method was used, which could assess the composition of microbiota until the species level. Second, the majority of the patients in this study had obesity, which can also affect the composition of Lactobacillus. It is known that Lactobacillus reuteri is abundant in obese patients, while Lactobacillus paracessei is abundant in nonobese patients. The pattern of a high-carbohydrate diet can also affect the composition of Lactobacillus [23-25].

In this study several things that affect the amount of microbiota have also been considered such as a history of antibiotic use, diet, age, and metabolic components. There are limitations to this study. The first is the number of samples, but the result can already answer the research questions, and the same number of samples was used as that in previous studies conducted abroad. The second is the difference in quantitative methods with RT-PCR, which only counts 3 types of genus level microbiota so that the exact compositions of other gut microbiota to the species level were not known.

\section{Acknowledgments}

We are grateful to all the staff in the Hepatobiliary Division, Department of Internal Medicine, Dr. Cipto Mangunkusumo Hospital, for supporting this research.

\section{Statement of Ethics}

All the patients gave written consent before stool collection. This study was approved by The Health Research Ethics Committee, Faculty of Medicine, University of Indonesia-Dr. Cipto Mangunkusumo Hospital (No. 684/UN2.F1/ETIK/2017).

\section{Disclosure Statement}

The authors have no conflicts of interest to declare.

\section{Author Contributions}

W.P.B. conducted the research study, database setting, data analysis, and wrote the manuscript. I.H. conceived the study idea, supervised the research process, and reviewed the 
manuscript. C.R.A.L. supervised and was involved in the research process, data analysis, and writing and editing the manuscript. I.R. assisted with the data analysis and statistics. R.A.G. supervised the entire research process and reviewed the study results and manuscript.

\section{Funding Sources}

This research was supported by grants from Hibah PITTA Universitas Indonesia.

\section{References}

1 Chalasani N, Younossi Z, Lavine JE, Diehl AM, Brunt EM, Cusi K, et al. The diagnosis and management of nonalcoholic fatty liver disease: practice Guideline by the American Association for the Study of Liver Diseases, American College of Gastroenterology, and the American Gastroenterological Association. Hepatology. 2012 Jun;55(6):2005-23.

2 Loomba R, Sanyal AJ. The global NAFLD epidemic. Nat Rev Gastroenterol Hepatol. 2013 Nov;10(11):686-90.

3 Hasan I. Perlemakan Hati Non Alkoholik. In: Alwi I, Setiati S, Setiyohadi B, Simadibrata M, Sudoyo A, editors. Buku Ajar Ilmu Penyakit Dalam. V. Jakarta: Interna Publishing; 2009. pp. 695-701.

4 Mikolasevic I, Orlic L, Franjic N, Hauser G, Stimac D, Milic S. Transient elastography (FibroScan(®)) with controlled attenuation parameter in the assessment of liver steatosis and fibrosis in patients with nonalcoholic fatty liver disease - Where do we stand? World J Gastroenterol. 2016 Aug;22(32):7236-51.

5 Sasso M, Beaugrand M, de Ledinghen V, Douvin C, Marcellin P, Poupon R, et al. Controlled attenuation parameter (CAP): a novel VCTE ${ }^{\mathrm{TM}}$ guided ultrasonic attenuation measurement for the evaluation of hepatic steatosis: preliminary study and validation in a cohort of patients with chronic liver disease from various causes. Ultrasound Med Biol. 2010 Nov;36(11):1825-35.

6 Chan WK, Nik Mustapha NR, Wong GL, Wong VW, Mahadeva S. Controlled attenuation parameter using the FibroScan ${ }^{\circledR}$ XL probe for quantification of hepatic steatosis for non-alcoholic fatty liver disease in an Asian population. United European Gastroenterol J. 2017 Feb;5(1):76-85.

7 Buzzetti E, Pinzani M, Tsochatzis EA. The multiple-hit pathogenesis of non-alcoholic fatty liver disease (NAFLD). Metabolism. 2016 Aug;65(8):1038-48.

8 Arslan N. Obesity, fatty liver disease and intestinal microbiota. World J Gastroenterol. 2014 Nov;20(44):16452-63.

9 Hawrelak JA, Myers SP. The causes of intestinal dysbiosis: a review. Altern Med Rev. 2004 Jun;9(2):180-97.

10 Mouzaki M, Comelli EM, Arendt BM, Bonengel J, Fung SK, Fischer SE, et al. Intestinal microbiota in patients with nonalcoholic fatty liver disease. Hepatology. 2013 Jul;58(1):120-7.

11 Wong VW, Tse CH, Lam TT, Wong GL, Chim AM, Chu WC, et al. Molecular characterization of the fecal microbiota in patients with nonalcoholic steatohepatitis—a longitudinal study. PLoS One. 2013 Apr;8(4):e62885.

12 Nobili V, Putignani L, Mosca A, Chierico FD, Vernocchi P, Alisi A, et al. Bifidobacteria and lactobacilli in the gut microbiome of children with non-alcoholic fatty liver disease: which strains act as health players? Arch Med Sci. 2018 Jan;14(1):81-7.

13 Boursier J, Mueller O, Barret M, Machado M, Fizanne L, Perez FA. dkk. The severity of nonalcoholic fatty liver disease is associated with gut dysbiosis and shift in the metabolic function of the gut microbiota. Hepatology. 2016 Mar;63(3):764-75.

14 Loomba R, Seguritan V, Li W, Long T, Klitgord N, Bhatt A, et al. Gut microbiomes-based metagenomics signature for non-invasive detection of advanced fibrosis in human non-alcoholic fatty liver disease. Cell Metab. 2017 May;25(5):1054-62.e5.

15 Furet JP, Firmesse 0, Gourmelon M, Bridonneau C, Tap J, Mondot S, et al. Comparative assessment of human and farm animal faecal microbiota using real-time quantitative PCR. FEMS Microbiol Ecol. 2009 Jun;68(3):351-62.

16 Wong VW, Vergniol J, Wong GL, Foucher J, Chan HL, Le Bail B, et al. Diagnosis of fibrosis and cirrhosis using liver stiffness measurement in nonalcoholic fatty liver disease. Hepatology. 2010 Feb;51(2):454-62. 


\section{Case Reports in Gastroenterology}

17 Chitturi S, Farrell GC, Hashimoto E, Saibara T, Lau GK, Sollano JD; Asia-Pacific Working Party on NAFLD. Non-alcoholic fatty liver disease in the Asia-Pacific region: definitions and overview of proposed guidelines. J Gastroenterol Hepatol. 2007 Jun;22(6):778-87.

18 Zhao Y, Wu J, Li JV, Zhou NY, Tang H, Wang Y. Gut microbiota composition modifies fecal metabolic profiles in mice. J Proteome Res. 2013 Jun;12(6):2987-99.

19 den Besten G, van Eunen K, Groen AK, Venema K, Reijngoud DJ, Bakker BM. The role of short-chain fatty acids in the interplay between diet, gut microbiota, and host energy metabolism. J Lipid Res. 2013 Sep;54(9):2325-40.

20 Puertollano E, Kolida S, Yaqoob P. Biological significance of short-chain fatty acid metabolism by the intestinal microbiome. Curr Opin Clin Nutr Metab Care. 2014 Mar;17(2):139-44.

21 Ma YY, Li L, Yu CH, Shen Z, Chen LH, Li YM. Effects of probiotics on nonalcoholic fatty liver disease: a metaanalysis. World J Gastroenterol. 2013 Oct;19(40):6911-8.

22 Tojo R, Suarez A. clemete MG, Reyes-Gavilan CG, Margolles A, Gueimonde M. Gut microbiota in health and disease: role of Bifidobacteria in gut hemostasis. World J Gastroenterol. 2014 Nov;20(41):15163-76.

23 van Best N, Jansen PL, Rensen SS. The gut microbiota of nonalcoholic fatty liver disease: current methods and their interpretation. Hepatol Int. 2015 Jul;9(3):406-15.

24 Ley RE. Obesity and the human microbiome. Curr Opin Gastroenterol. 2010 Jan;26(1):5-11.

25 Milion M, Maraninchi M, Henry M, et al. Obesity associated gut microbiota is enriched in Lactobacillus reuteri and depleted in Bifodbacterium animalia and Methanobrevivacter smithii. Int J Obes (Lond). 2012 Jun;36(6):817-25.

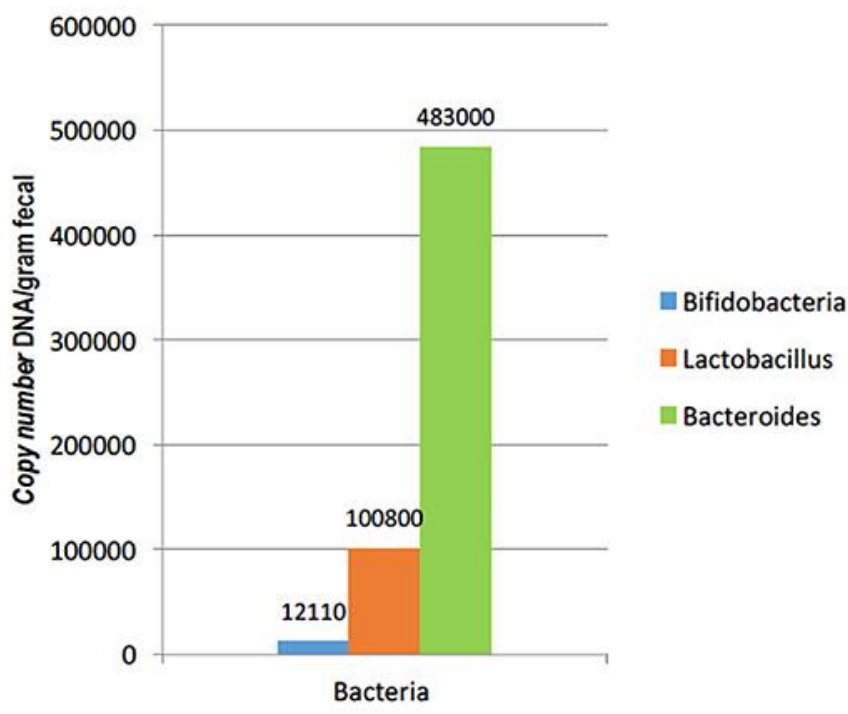

Fig. 1. Proportion of gut microbiota in NAFLD patients. 
Table 1. Characteristics of study subjects

\begin{tabular}{|c|c|c|c|}
\hline Variables & $\begin{array}{l}\text { Total } \\
(n=60)\end{array}$ & $\begin{array}{l}\text { Nonsignificant } \\
\text { fibrosis (F0-F1) } \\
(n=44)\end{array}$ & $\begin{array}{l}\text { Significant } \\
\text { fibrosis (F2-F4) } \\
(n=16)\end{array}$ \\
\hline \multicolumn{4}{|l|}{ Gender, $n(\%)$} \\
\hline Male & $28(46.7)$ & $20(71.4)$ & $8(28.6)$ \\
\hline Female & $32(53.3)$ & $24(75)$ & $8(25)$ \\
\hline Age (mean \pm SD), years & $56(10.21)$ & $55.6(10.46)$ & $58(9.2)$ \\
\hline \multicolumn{4}{|l|}{ Diabetes mellitus, $n(\%)$} \\
\hline Yes & $51(85)$ & $38(74.5)$ & $13(25.5)$ \\
\hline No & $9(15)$ & $6(66.7)$ & $3(33.3)$ \\
\hline \multicolumn{4}{|l|}{ Dyslipidemia, $n(\%)$} \\
\hline Yes & $35(58.3)$ & $26(74.3)$ & $9(25.7)$ \\
\hline No & $25(41.7)$ & $18(72)$ & $7(28)$ \\
\hline \multicolumn{4}{|l|}{ Obesity (IMT $\geq 25), n(\%)$} \\
\hline Yes & $41(68.3)$ & $27(65.9)$ & $14(34.1)$ \\
\hline No & $19(31.6)$ & $17(89.5)$ & $2(10.5)$ \\
\hline \multicolumn{4}{|l|}{ Central obesity, $n(\%)$} \\
\hline Yes & $57(95)$ & $41(71.9)$ & $16(28.1)$ \\
\hline No & $3(5)$ & $3(100)$ & $0(0)$ \\
\hline Body mass index (mean $\pm \mathrm{SD}$ ), $\mathrm{kg} / \mathrm{m}^{2}$ & $27(4.2)$ & $26.4(3.9)$ & $29.9(3.65)$ \\
\hline Triglycerides (mean \pm SD), mg/dL & $138.8(60.91)$ & $137.8(63.08)$ & $135.8(59.36)$ \\
\hline $\mathrm{HbA1c}($ mean $\pm \mathrm{SD}), \%$ & $7.1(1.87)$ & $6.7(1.44)$ & $8(2.52)$ \\
\hline $\operatorname{AST}($ mean \pm SD), U/L & $21.83(9.77)$ & $20.3(5.50)$ & $27.5(16.92)$ \\
\hline $\operatorname{ALT}($ mean $\pm \mathrm{SD}), \mathrm{U} / \mathrm{L}$ & $23(11.51)$ & $21.7(10.10)$ & $28.2(15.81)$ \\
\hline
\end{tabular}

SD, standard deviation; AST, aspartate aminotransferase; ALT, alanine aminotransferase.

Table 2. Comparison of the proportion of gut microbiota between the nonsignificant liver fibrosis group and the significant liver fibrosis group

\begin{tabular}{llll}
\hline Species & $\begin{array}{l}\text { Total } \\
(n=60)\end{array}$ & $\begin{array}{l}\text { Nonsignificant } \\
\text { fibrosis (F0-F1) } \\
(n=44)\end{array}$ & $\begin{array}{l}\text { Significant } \\
\text { fibrosis (F2-F4) } \\
(n=16)\end{array}$ \\
\hline Bifidobacteria & $\begin{array}{l}76.5 \\
(0-330,000)\end{array}$ & $\begin{array}{l}95 \\
(0-330,000)\end{array}$ & $\begin{array}{l}22 \\
(0-2,800)\end{array}$ \\
\hline Lactobacillus & $\begin{array}{l}2,800 \\
(0-910,000)\end{array}$ & $\begin{array}{l}2,050 \\
(0-910,000)\end{array}$ & $\begin{array}{l}7,000 \\
(6.3-100,000)\end{array}$ \\
\hline Bacteroides & $\begin{array}{l}130,000 \\
(41-1,200,000)\end{array}$ & $\begin{array}{l}73,000 \\
(41-880,000)\end{array}$ & $\begin{array}{l}310,000 \\
(5,400-1,200,000)\end{array}$ \\
\hline
\end{tabular}

Values are median DNA copy number/gram feces (minimum-maximum). 